\title{
USABILIDADE DE SITES E GESTÃO DA INFORMAÇ̃̃O NA ANÁLISE E DIVULGAÇÃO DOS PROGRAMAS DE RECREAÇÃO EM SITES DE RESORTS NO BRASIL
}

Recebido em: 19/10/2018

Aceito em: 15/05/2019

\author{
Marília Amábile Guarizo ${ }^{1}$ \\ Gisele Maria Schwartz ${ }^{2}$ \\ Universidade Estadual Paulista (UNESP) - Campus de Rio Claro \\ Rio Claro - SP - Brasil
}

\begin{abstract}
RESUMO: Esse estudo qualitativo teve por objetivos analisar os sites de resorts, com base na gestão da informação e na usabilidade de sites, bem como, investigar a divulgação dos programas de recreação. Por meio de pesquisa exploratória foram elencados os sites, os quais foram avaliados com base nas Teorias da Gestão da Informação e da Usabilidade de sites, criando-se categorias a priori, para análise dos dados. Os resultados indicam que as informações acerca do programa de atividades recreativas oferecidas são precárias e pouco motivam a adesão à prática. Além disso, os sites dos resorts não atendem a todos os critérios de usabilidade, dificultando a busca por informações pelo usuário, podendo gerar aos estabelecimentos o comprometimento das vendas, devido a essas falhas existentes nos sites. Sugerem-se novos estudos relacionando as temáticas envolvendo tecnologias, turismo, recreação e lazer.
\end{abstract}

PALAVRAS-CHAVE: Atividades Recreativas. Gestão da Informação. Usabilidade de Sites.

\section{USABILITY OF WEBSITES AND INFORMATION MANAGEMENT IN THE ANALYSIS AND DISSEMINATION OF RECREATION ON RESORT SITES IN BRAZIL}

ABSTRACT: This qualitative study aimed to analyzing resorts sites, based on information management and sites usability and also investigated the diffusion of the recreation program. An exploratory research was conducted aiming to investigating those sites, which were evaluated based on Information Management and Usability of sites Theories. A priori categories for data analysis were created and results indicate precariousness information about the offered recreation activities do not motivate the adherence to the practice. In addition, resorts' websites do not meet all of the usability criteria, making it difficult for users to search for information, which may lead to

\footnotetext{
${ }^{1}$ Mestre em Desenvolvimento Humano Tecnologias. Membro do LEL - Laboratório de Estudos do Lazer, DEF/IB/ UNESP/RC.

${ }^{2}$ Doutora em Psicologia Escolar e do Desenvolvimento (IPUSP). Docente no Depto. De Educação Física/IB/UNESP/RC. Orientadora nos cursos de Mestrado e Doutorado, nos PPG em Ciências da Motricidade e em Desenvolvimento Humano e Tecnologias. Líder do LEL - Laboratório de Estudos do Lazer, DEF/IB/ UNESP/RC.
} 
sales compromises due to such site failures. New studies are suggested relating to these themes involving technologies, tourism, recreation and leisure.

KEYWORDS: Recreational Activities. Information Management. Usability of Websites.

\section{Introdução}

As atividades recreativas, assim como os espaços e equipamentos voltados ao lazer, estão presentes em muitos hotéis. Entre eles, pode-se destacar, principalmente os resorts, visto que estes são voltados especificamente para o lazer, como pode ser percebido na definição do Ministério do Turismo ( BRASIL, 2014): "Hotel com infraestrutura de lazer e entretenimento que disponha de serviços de estética, atividades físicas, recreação e convívio com a natureza no próprio empreendimento.”.

Este tipo de hospedagem referente aos resorts, geralmente, é usufruída no contexto do lazer. No que se refere ao lazer, este pode ser entendido, como o tempo disponível no qual o indivíduo pode escolher, de forma livre, por ocupações, as quais possibilitem repouso, divertimento ou desenvolvimento, após o cumprimento de suas obrigações relacionadas ao trabalho, à família ou ao âmbito social (DUMAZEDIER, 1979). Existem diversas possibilidades de se ocupar o tempo destinado ao lazer, seja com momentos de descanso, ou, ainda, com diversos tipos de atividades recreativas. A recreação pode ser entendida como atividades realizadas no tempo dedicado ao lazer, com o intuito de atender às vontades e anseios dos participantes (BRUHNS, 1997). Para Guerra (1985), o lazer está associado ao tempo que uma pessoa destina a si própria, tendo uma atitude favorável ao usufruto qualitativo do tempo disponível e a recreação se refere às atividades realizadas de forma espontânea, as quais possibilitam prazer e momentos de criação. Entre as diferentes formas de se recrear encontram-se aquelas desenvolvidas nos espaços específicos dos resorts, com suas 
peculiaridades e diferenciações.

Os resorts, para Rodrigues e Dantas (2017) podem ser considerados como elementos autocontidos, nos quais os hóspedes satisfazem muitas de suas necessidades de descanso, entretenimento e diversão no próprio estabelecimento, sem precisar se deslocar para outro lugar. Entretanto, para que isto efetivamente ocorra, há necessidade de uma programação bastante variada, para atender às diversas expectativas dos hóspedes.

Em geral, ao fazer a opção por um meio de hospedagem em uma viagem, as principais influências são referentes às questões de custo-benefício (DHAMI; DENG, 2017) e a possibilidade de vivências prazerosas (JAHREN; SUI, 2017). Desta forma, as informações acerca dos espaços e equipamentos de lazer e também sobre as atividades desenvolvidas, seja para crianças, adolescentes, adultos ou idosos, podem fazer parte dos aspectos determinantes na escolha de um destino. Sendo assim, divulgar adequadamente as opções de recreação oferecidas nesse âmbito do lazer, pode representar um diferencial importante para um resort.

Nesse sentido, ao desenvolver um site, esses fatores referentes à programação de recreação e lazer, bem como, aos espaços e equipamentos, devem merecer destaque, visando atrair a atenção do usuário. Para tanto, é necessário que o site seja bem elaborado, tenha destaque para os links voltados às opções no lazer, tenha harmonia de cores e seja funcional, de modo a ser receptivo para todos os públicos e com letras de fácil visualização, para que, mesmo aqueles com a acuidade visual diminuída, consigam enxergar com nitidez e, possam ter melhor compreensão do conteúdo.

Todos esses requisitos, referentes à qualidade de um site e à difusão correta da informação apresentada, podem ser facilmente alcançados se, durante a construção de 
um site, os requisitos básicos da usabilidade de sites forem levados em consideração, visando garantir que a interação do usuário com a interface se dê com qualidade, de forma rápida e fácil (RODRÍGUEZ et al., 2017). A teoria da usabilidade de sites pode ser entendida como "[...] medida na qual um produto pode ser usado por usuários específicos para alcançar objetivos específicos com eficácia, eficiência e satisfação em um contexto específico de uso." (ABNT, 2002).

Dentre as possibilidades de avaliação da usabilidade de um site pode-se citar a proposta de avaliação heurística de Nielsen (2000), a qual visa garantir que a interação do usuário em uma interface contenha qualidade, facilidade e rapidez. Este método é um dos mais utilizados para se mensurar a usabilidade de sites (PAZ et al. 2018).

Outro fator de grande importância para que um site dialogue diretamente com o usuário, refere-se à disposição e qualidade das informações veiculadas no ambiente virtual. Nesse sentido, os pressupostos da teoria da gestão da informação podem auxiliar no processo de escolha daquilo que deve estar em destaque, assim como, na elaboração dos textos explicativos, para que o usuário tenha total compreensão da mensagem.

A gestão da informação pode ser entendida como a soma de estratégias utilizadas para reconhecer, coletar e mapear informações relevantes, visando dar suporte nas tomadas de decisão (SCHWARTZ et al., 2010). Corroborando a autora, Pinto (2015) aponta que, no processo da Gestão da Informação, deve ocorrer a concepção, implementação e desenvolvimento de processos os quais são pertinentes ao fluxo de informações, para que se possam construir modelos operacionais eficientes e rentáveis. Focalizando o âmbito da hospitalidade, pode-se dizer que a gestão da informação se dá com base em serviços. 
Para Borges Filho et al. (2017), os serviços prestados no campo da hospitalidade se restringem a períodos determinados, correspondente apenas ao tempo de hospedagem do cliente. Por outro lado, as facilidades advindas da utilização de tecnologias avançadas e da internet, podem se apresentar como uma importante estratégia de marketing (BUGDAY; LEMBET, 2016), auxiliando na venda e, inclusive, na obtenção de clientes por meio da divulgação dos estabelecimentos, tanto da estrutura geral, dos espaços destinados ao lazer, assim como, das atividades recreativas ofertadas. Nesse sentido, a teoria da gestão da informação pode auxiliar no processo de escolha daquilo que deve estar em destaque, bem como, na elaboração dos textos explicativos constantes em um site, para que este seja atrativo, objetivo e alcance o usuário de forma assertiva, expondo os pontos principais e despertando o interesse de se hospedar no estabelecimento.

No entanto, ainda que estas teorias já sejam conhecidas e validadas, ao se voltar a atenção para os âmbitos do turismo e do lazer, pouco se sabe sobre a difusão das atividades ofertadas por resorts, já que esse nicho do mercado tem sido pouco explorado na literatura acadêmica, mesmo representando uma importante estratégia de divulgação e venda dos serviços desses estabelecimentos. Além disso, no que se refere aos sites desses resorts, nem sempre os aspectos relativos às teorias da usabilidade de sites e da gestão da informação são levados em consideração. Esses fatores podem acarretar prejuízos, de um lado para os resorts, os quais podem ter a venda comprometida, devido à falta de gerenciamento apropriado de informações e, também, pelo funcionamento inadequado, ou pela ausência de atratividade de seu site. Por outro lado, o usuário, o qual pode ser considerado um possível cliente, pode sofrer perdas em relação às escolhas que pode fazer para ocupar seu tempo disponível, o que pode influenciar até no gerenciamento do seu próprio tempo e no preparo adequado 
para praticar certas atividades, como por exemplo, não colocar na bagagem roupas adequadas à prática de atividades físicas, por não saber que elas seriam oferecidas.

Com base no exposto, torna-se importante verificar como os programas contendo as opções de atividades recreativas têm sido divulgados nos sites de resorts, de modo a diagnosticar se esses sites são atrativos, organizados, com informações adequadas e esclarecedoras. Diante disso, esse estudo tem por objetivos analisar os sites de resorts, com base na gestão da informação e na usabilidade de sites, bem como, investigar a divulgação dos programas de recreação.

\section{Método}

O presente estudo teve uma natureza qualitativa, tendo em vista que este método se adequa aos objetivos propostos. De acordo com Richardson (2017), a pesquisa qualitativa possibilita descrever, de forma abrangente a complexidade do problema, resultando em maior compreensão do universo pesquisado. Para Torrance (2017), as técnicas utilizadas neste método proporcionam reflexões mais densas e aprofundadas.

O estudo foi desenvolvido por meio da união de pesquisas bibliográfica e exploratória. Para a pesquisa bibliográfica, foram consultadas fontes provenientes de artigos, livros, teses e dissertações referentes às temáticas do estudo. Para a pesquisa exploratória, o site da Associação Brasileira de resorts - ABR foi utilizado para a seleção dos resorts. Foram identificados 48 associados, os quais estão distribuídos em 13 estados brasileiros e abrangem as 5 regiões do país. Para selecionar os sites para análise, a qual ocorreu com base na gestão das informações e na usabilidade de sites, foi feita a identificação de quais apresentavam informações acerca do programa de atividades recreativas disponibilizado aos clientes durante a estadia. 
Para a análise dos sites, foram utilizadas como base a Teoria da Gestão da Informação e a Teoria da Usabilidade de sites. Para análise dos dados provenientes do diagnóstico e da avaliação dos sites, foi utilizada a técnica de Análise de Conteúdo (BARDIN, 2016) a qual prevê a organização dos dados em categorias. Neste estudo, essas categorias foram estabelecidas a priori. Com base na Teoria da Usabilidade de sites foram levados em consideração 3 categorias para análise dos dados: A conteúdo, B - layout, C - operacionalização, os quais buscam abranger os diversos elementos da proposta de avaliação heurística (NIELSEN, 1999). Baseado na Teoria da Gestão da Informação, as categorias de análise dos dados foram: 1- Identificação dos tipos de atividades ofertadas, 2- Clareza e facilidade de compreensão das informações, 3- Atualização dos dados.

\section{Resultados e Discussão}

Ao buscar pelos sites dos resorts, um não foi encontrado, sendo que a divulgação, neste caso, era realizada por meio do site de uma Central de Operadoras de Turismo, a qual, aparentemente, era responsável por agenciar o estabelecimento. Este site, portanto, foi excluído. Partiu-se, então, para a busca de informações acerca do programa de atividades recreativas nos demais sites. Dos 47 restantes, 1 não apresentava as informações buscadas. Restaram, então, 46 sites a serem analisados, destes, todos apresentavam atividades recreativas, contudo, apenas 3 divulgavam informações mais detalhadas acerca da programação oferecida, sendo, portanto, selecionados para análise.

A fim de preservar os dados dos resorts e garantir-lhes privacidade, sigilo e confidencialidade, os estabelecimentos foram renomeados de R1, R2 e R3, sendo que 
os resorts $\mathrm{R} 1$ e R2 pertencem a uma mesma rede hoteleira ${ }^{3}$ e o R3 faz parte de outro grupo. Após a seleção e renomeação, realizou-se a análise com base nas teorias da Gestão da Informação e da Usabilidade de sites.

\section{Análise com Base na Teoria da Usabilidade de Sites}

No que se refere à categoria A- conteúdo do site, percebeu-se que o modo como as informações acerca do programa de atividades recreativas estava veiculado nos sites não favorece a apreensão dos conteúdos. Os resorts R1 e R2, pertencentes à mesma rede, apresentavam informações de forma parcial, contendo apenas atividades principais, com uma breve descrição. Já R3 apresentava a programação dos 7 dias da semana, de cada faixa etária, porém, não havia descrição das atividades, constando apenas o horário, o nome da atividade e o local.

Desta forma, os conteúdos, disponíveis nos sites dos resorts R1 e R2 apresentaram mais informações acerca do conteúdo das atividades, embora não informassem detalhes sobre horário e local das atividades. Já no site do R3, faltou esclarecimento mínimo acerca do que se tratava cada atividade. Outro fator percebido em ambos os sites foi que, ao se clicar em um ícone, este apresentava uma marcação, a qual possibilitava identificar em qual link estavam as informações que se estava lendo. No entanto, os ícones não ficavam demarcados após a visualização.

Focalizando a faixa etária, os resorts R1 e R2, apresentavam atividades para crianças de 4 a 12 anos, divididas em dois grupos, ( 4 a 6 e 7 a 12 anos), teens, correspondente a adolescentes e adultos. O outro resort apresentava mais divisões para a faixa etária das crianças, sendo elas: 3 a 5 anos, 6 a 8 anos, 10 a 12 anos e, mais 2 divisões, correspondentes a adolescentes e adultos.

\footnotetext{
${ }^{3}$ Rede hoteleira: grupo de hotéis, próprios ou arrendados, administrados por um grupo hoteleiro (BRASIL, 2016).
} 
A falta de esclarecimento nos sites, acerca das atividades a serem vivenciadas, pode acarretar prejuízos para os resorts, já que o entendimento das atividades é de suma importância para catalisar a motivação para a participação. Para tanto, é necessário informar de forma clara e legível, os objetivos e as exigências para se realizar uma atividade. Esses fatores podem impactar na escolha de uma atividade. Logo, a divulgação dessas informações atendendo a critérios de legibilidade e credibilidade, contribui para que a mensagem seja, de fato, benéfica e útil ao usuário (VIVEKANANTHAM et al., 2017)

No que se refere à categoria B-layout, pode-se notar, em todos os sites, que as cores parecem estar harmonizadas, entretanto as letras, de modo geral, não são bem visíveis e o formato não é coerente com a proposta de leitura. Em alguns ícones, pode-se perceber excesso de textos e poucas imagens, sobretudo no site do R3, o que pode afetar a estética e atrapalhar a visualização adequada da informação, comprometendo, inclusive, a atratividade. Para Uribe, Álvarez e Menéndez (2017), entre os aspectos que indicam a qualidade de um site, o layout se apresenta como um fator bastante influente. Sendo assim, nota-se a necessidade de adequação e melhoria deste quesito, em todos os sites analisados.

Quanto às estruturas dos sites, os resorts R1 e R2, apresentavam a mesma configuração, apenas adequando os conteúdos para descrever as estruturas e serviços para cada resort. O ícone o qual continha informação sobre as estruturas e atividades era denominado, Lazer e facilidades. De forma geral, em todos os sites pode-se notar a harmonia entre a quantidade de texto e imagens apresentadas, fazendo com que não houvesse excesso de informações. Esses fatores podem favorecer a compreensão dos conteúdos apresentados, visto que os requisitos relacionados a estética do site apresentam relação direta com aspectos necessários inerentes à clareza 
de informações (CAETANO et al., 2016). Porém, o tamanho das letras utilizadas nos sites dos resorts não facilita a visualização, além disso, não havia destaque nas partes principais.

No site do R3, havia 3 ícones destinados às informações acerca das estruturas e atividades do contexto do lazer, eram eles: Atividades, Monitoria e Programação. Em alguns ícones do site, percebe-se excesso de texto e poucas imagens, o que pode favorecer o desinteresse do usuário, fazendo com que este não tenha vontade em despender mais tempo para buscar novas informações, ou para voltar ao site. Tendo em vista que as questões visuais de um site podem favorecem o envolvimento emocional e a intenção do usuário de voltar a visitar o site (MARASCO et al., 2018), o equilíbrio entre imagens e figuras poderia contribuir para a conquista desse usuário. $\mathrm{P}$, o site desse resort pode obter prejuízos, pela escassez de imagens.

No que se refere à categoria C- operacionalização, foram analisados os suportes disponíveis nos sites para facilitar a navegação do usuário. Nos três sites dos resorts, pode-se perceber clareza nos menus e links, assim como, notou-se que havia destaque para aqueles links que continham informações sobre as formas de contatar o estabelecimento. No entanto, algumas informações relevantes para o usuário estavam em locais de pouco destaque, dificultando o acesso a eles. No R3, por exemplo, alguns links foram encontrados no final da página, em ícones pequenos, com pouco realce. Eram eles, referentes ao mapa do hotel, o qual possibilitava visão geral do estabelecimento, o mapa no qual era possível traçar a rota do seu local até os resorts e, também, os vídeos institucionais.

A dificuldade em localizar os conteúdos, identificada nos sites analisados, pode refletir no tempo necessário para navegar nesse site (MARTEL, 2017) e na conquista do usuário, já que este requer que seja possível encontrar as informações 
desejadas com facilidade, não desperdiçando seu tempo. Nesse sentido, Jacoski, Dallacorte e Mota (2017) afirmam que um usuário passa apenas cerca de dois minutos fazendo buscas em um site, desta forma a sua fidelização deve ocorrer nesse pequeno espaço de tempo. Caso o usuário não encontre aquilo que busca, este passará a procurar por outro site. Por conseguinte, a facilidade de operacionalização, sobretudo ao que se refere ao acesso à informação e, especialmente, um modo de cativar o usuário, devem ser priorizados (SIMÃO et al., 2016).

Em todos os sites analisados, pode-se perceber que as opções de contato estavam em evidência, inclusive, constavam diferentes canais, como por exemplo, telefone, redes sociais, email ou chat. Portanto, as formas de esclarecimentos de dúvidas, consultas ou reservas apresentavam-se com o destaque necessário, não trazendo prejuízos aos resorts. Além disso, no site do R3 notou-se a presença de links visualizados por meio de figuras, os quais podem facilitar a navegação e o acesso a informações. Notou-se, ainda, a preocupação em destacar itens relacionados à operacionalização, como menus, canais de ajuda ou contato, mecanismos de buscas, entre outros, os quais podem auxiliar na motivação do usuário para continuar a navegar pelo site (LONGARAY et al., 2017) e podem ter impacto direto na satisfação do usuário (COSTA; VIDOTTI, 2017).

Em nenhum dos sites se notou a presença de adaptações no ambiente, as quais pudessem facilitar a utilização por pessoas idosas, ou com comprometimentos visuais ou auditivos. Essa falta de adaptação é evidenciada no estudo de Hussain, Ross e Bednar (2018), no qual participantes relataram dificuldades referentes às questões de acessibilidade, usabilidade e design em sites. Tais limitações podem estar relacionadas, por exemplo, ao comprometimento da acuidade visual, ou na coordenação de movimentos (JONGSTRA et al., 2017). No que tange aos deficientes 
visuais, estes podem apresentar maior dificuldades em navegar pelos sites, sendo assim, há necessidade de adaptar as interfaces para que o acesso às informações por esse público seja facilitado (LAZAR; OLALERE; WENTZ, 2012).

O ambiente virtual deve permitir a utilização por populações especiais, para se garantir a inclusão de todos os cidadãos e a recepção de informações (SUTTON, 2017). Por conseguinte, identificou-se que os sites analisados devem atentar para diminuir as dificuldades de navegação e adequar o tamanho das letras, com o intuito de atender às pessoas com restrições ou limitações.

\section{Análise com Base na Teoria da Gestão da Informação}

Em relação à categoria 1 - Identificação dos tipos de atividades ofertadas, nos sites de R1 e R2, identificou-se no programa de recreação e lazer, atividades em comum para adultos e crianças, entre elas: atividades artísticas como, shows diversos (comédia, teatro de fantoches, circo, palhaço), oficinas gastronômicas e de artesanato, discoteca para família, sessão cinema, atividades esportivas, atividades de aventura (rapel, escalada, tirolesa), atividades aquáticas, atividades circenses, sinuca com os pés e atividades utilizando exergames e videogame. Notou-se que, entre as atividades elencadas, existe, inclusive, a utilização de jogos eletrônicos no campo da recreação, o que pode ser considerada uma novidade, já que em resorts, costuma-se valorizar a presença de espaços naturais.

No campo educacional, a utilização de games, mais particularmente os exergames, também já são utilizados como ferramentas pedagógicas (SOUZA et al., 2017). Para Oliveira (2017) os exergames auxiliam na obtenção e no aprimoramento de habilidades diversificadas, de forma lúdica e prazerosa. Já no campo da saúde, podem auxiliar na disseminação do conhecimento acerca da prevenção de doenças 
(BARBOSA, 2013; RODRIGUES, 2014; DIAS, 2015). Alguns autores também se dedicaram a estudar ou elaborar games utilizados para difundir conhecimentos acerca de doenças e estratégias de prevenção, como, por exemplo, a dengue (NUNES, 2014; LIMEIRA, 2015; PAIM, 2015; SPARAPANI, 2015).

Associando os campos da Educação e da Saúde, Vaghetti (2013) desenvolveu um estudo relacionando os exergames com a Educação Física, reafirmando sua importância no incentivo à prática de atividades físicas. Todos os aspectos abordados por esses e outros estudos, podem ser levados em consideração, também no contexto dos resorts, promovendo ações educativas, por intermédios da oferta de atividades utilizando games, com ou sem movimento, resultando em vivências significativas e educativas, as quais poderão ter reflexos no cotidiano dos participantes.

Com o foco nos públicos envolvendo adolescentes e adultos, foram citadas atividades como, trilhas e caminhadas, hidroginásticas, aulas de ginásticas e ritmos, noites dançantes, música ao vivo, gincanas e desafios, paintball, atividades esportivas (vôlei, futebol, futebol society, corrida, tênis, boliche), clínica de esportes, bingo, jogos de mesa, betes, jogos de terror, festas temáticas, aulas de automaquiagem, workshop de vinhos para adultos, palestras e oficinas gastronômicas. Especificamente para crianças os sites apresentavam personagens, piquenique, brinquedos, caças temáticas, jogos noturnos, passeios (ecológicos, ao mini-zoo, à horta e ao pomar), artesanato kids, jogos de salão (socialização).

Em todas as faixas etárias, a presença de atividades físicas associadas aos aspectos lúdicos pode representar um potencial para desenvolver trabalhos de conscientização acerca da importância da adoção de um estilo ativo e saudável, assim como, para promover a socialização entre os participantes, a aquisição de novas habilidades e novos conhecimentos, nos momentos destinados ao lazer. Os estudos de 
Noronha e Ribeiro (NORONHA; RIBEIRO, 2017) apontam que, por meio de atividades lúdicas, podem-se transmitir conhecimentos acerca da conquista de uma vida mais saudável.

Entre as vivências elencadas nos programas analisados, notou-se a presença de atividades nas quais os aspectos pedagógicos poderiam estar presentes, para além da diversão, como, por exemplo, os passeios ou trilhas ecológicas e as aulas de gastronomia. Os estudos de Pereira et al. (2013) apontam o elemento lúdico como um importante recurso para se trabalhar o conceito de preservação ambiental. Nas aulas de gastronomia, é possível, por meio do brincar, utilizando elementos lúdicos como os jogos, facilitar a compreensão das crianças acerca da diferenciação de alimentos saudáveis e não saudáveis, o que pode resultar na melhoria da alimentação desse público (NORONHA; RIBEIRO, 2017). No entanto, embora tais atividades constem no programa de atividades recreativas dos sites dos resorts, não há evidencias da exploração dos elementos pedagógicos inerentes às atividades.

No que tange à categoria 2 - Descrição das atividades recreativas, nas informações expostas nos sites dos resorts R1 e R2, pode-se perceber a existência de uma breve explicação de cada uma das atividades, as quais possibilitavam ao usuário o entendimento do que se tratava cada atividade. Por outro lado, não era possível identificar para qual faixa etária estas vivências eram especificamente direcionadas. As atividades eram agrupadas em crianças e adolescentes e adultos, de modo que não era possível afirmar se todas as atividades eram ofertadas para todas as faixas etárias, ou se havia algum tipo de separação.

A linguagem utilizada era clara, objetiva e concisa, porém, não estavam claros os dias, horários e locais nos quais as atividades eram ofertadas. Era possível identificar características das atividades, o que facilitava a compreensão do conteúdo 
e, também, elementos da atividade, identificando quais se tratavam, por exemplo, de atividades físicas, manuais ou esportivas, ou ainda, de shows. Pode-se perceber a inadequação do conteúdo em relação à denominação do ícone no site do R3, pois, havia um ícone denominado Cavalgada, o qual reunia informações acerca da fazendinha em geral, não somente à cavalgada.

Caso não haja clareza na elaboração dos conteúdos expressos, a mensagem pode ficar truncada, ou mesmo não atender às expectativas do usuário. A definição de estratégias adequadas de comunicação pode favorecer a melhoria do envolvimento dos usuários aos sites, tendo em vista, a possibilidade de oferecer maior consistência e aproximação com os interesses durante a busca de informações (ZHANG; CHOI, 2018).

A facilidade de compreensão das informações advindas de sites representa um fator de grande relevância em todos os setores da vida social. Estudos atualizados reiteram a importância da combinação de diversas estratégias para imprimir maior clareza e facilidade de compreensão das informações veiculadas online. Além disso, esses estudos evidenciam o valor dessas abordagens combinadas, com o intuito de favorecer a legibilidade da informação, baseada, inclusive, no conteúdo emocional, conforme salientam Denecke e Deng (2015) e Beaunoyer et al. (2017).

No que se refere à categoria 3- Período de oferta das atividades, nos sites dos resorts $\mathrm{R} 1$ e R2 havia a possibilidade de se baixar do próprio site a programação por mês, deixando claro, ao usuário, o período no qual este poderia encontrar tais atividades nos resorts. Já no site do R3, apenas estavam explícitos o horário, o nome da atividade e o local, no entanto, não constavam os dias ou períodos nos quais as atividades eram ofertadas, o que impedia o hóspede de ter o entendimento mínimo acerca da realização de cada atividade. Além disso, não era possível identificar se 
aquela programação era válida para todos os meses do ano, ou apenas para um período específico, pois não constavam informações acerca do período no qual as atividades eram desenvolvidas.

O conteúdo pode ser considerado um importante fator no que tange às estratégias de comunicação e avaliação de produtos ou mensagens, podendo impactar nas decisões a serem tomadas pelo usuário, quando for o caso (SELLER; LAURINDO, 2018). Além disso, a desatualização dos conteúdos dos programas expressos nos sites pode trazer prejuízo para os resorts, carecendo, assim, de revisão e atualização por parte desses estabelecimentos.

Os investimentos em comunicação são imprescindíveis para aumentar o alcance de clientes. Uma das possibilidades para o fortalecimento do canal de comunicação consiste em utilizar estratégias de marketing voltadas ao relacionamento com o cliente, o que pode resultar em impactos positivos nas vendas (SCHNEIDER; ZILLI; VIEIRA, 2017).

A promoção da ligação do cliente com o conteúdo requer atenção acerca, entre outros fatores, da atualidade dos dados. A experiência agradável e que atenda aos anseios dos usuários, amplifica a adesão do usuário, bem como, intensifica a possibilidade de recomendação do acesso, garantindo assim, maior divulgação e credibilidade às informações contidas nos sites (LEE, 2018).

\section{Conclusão}

Com base nos resultados encontrados após a análise dos sites, notou-se que diversas são as possibilidades de atividades praticadas no tempo destinado ao lazer, inclusive, durante a estadia em resorts. Entretanto, embora existam aspectos que contribuam para disseminação do conhecimento acerca das possíveis atividades 
recreativas para ocupar o tempo disponível, estes não são apresentadas nos sites de forma a contribuir para a divulgação dos resorts. As informações são primordiais para se difundir as possibilidades de vivencias prazerosas no âmbito do lazer, aspecto que merece atenção no desenvolvimento dos sites dos resorts analisados.

Além disso, pode-se perceber a existência de lacunas a serem preenchidas, no que se refere aos sites dos resorts, os quais não atendem a todos os critérios de usabilidade, sobretudo, quanto ao conteúdo das informações e ao layout adequado para pessoas com alguma dificuldade visual. A inadequação dos sites traz prejuízos aos usuários, no momento das buscas por informações e para o próprio estabelecimento, tendo em vista que as vendas podem ser comprometidas, devido às falhas existentes nos sites.

Entre as limitações do presente estudo, pode-se citar o restrito número de resorts analisados, já que foi utilizada apenas uma fonte para busca dos mesmos. A ampliação para outras bases de busca, talvez possa acrescentar novos resorts em futuros estudos. Outra limitação do presente estudo pode ser a ausência da coleta de opinião de usuários desses sites de resorts, no que tange às informações sobre as atividades recreativas. A coparticipação do público nas pesquisas pode auxiliar na complementação dos dados e ampliar a fidedignidade dos dados. Como sugestão para futuros estudos, indica-se a investigação sobre a influência das atividades praticadas no tempo disponível para a adoção de hábitos mais saudáveis, como mudanças do comportamento alimentar e prática sistematizada de atividades físicas.

\section{REFERÊNCIAS}

ASSOCIAÇÃO BRASILEIRA DE NORMAS TÉCNICAS. NBR 9241-11: requisitos ergonômicos para trabalho de escritório com computadores: parte 11 - orientações sobre usabilidade. Rio de Janeiro: ABNT, 2002. 
BARBOSA, C.D.P. Análise da resposta da frequência cardíaca de adultos jovens saudáveis durante performance em um jogo de realidade virtual de imersão. $73 \mathrm{f}$. Dissertação (Mestrado em Terapia Ocupacional): Departamento de Terapia Ocupacional, Universidade Federal de São Carlos, São Carlos, 2013.

BARDIN, L. Análise de Conteúdo. São Paulo: Edições, 2016.

BEAUNOYER, E. et al. Understanding online health information: Evaluation, tools, and strategies. Patient education and counseling, Netherlands v.100, n.2, p. 183$189,2017$.

BORGES FILHO, C. C.; REINHARDT, M. P. S.; CARVALO, V. M.; ANJOS, S. J. G. A gestão da informação como subsídio para tomada de decisão: estudo de caso dos hotéis em Balneário Piçarras/SC. Applied Tourism, Balneário Camboriú,v.2, n.1, p. 40-51, 2017.

BRASIL. Ministério do Turismo. Internet é Fonte para 1,87 milhão de Turistas Estrangeiros, 2014. Disponível em: https://www.turismo.gov.br/turismo/noticias/todas_Acesso em: 05 dez. 2017.

. Ministério do Turismo. Regulamento do sistema oficial de classificação de meios de hospedagem. Brasília: Ministério do Turismo, 2011. Disponível em: https://www.classificacao.turismo.gov.br/MTUR-classificacao/mtur-site/ Acesso em: 05 dez. 2017.

. Ministério do Turismo. Glossário do Turismo, 2016. Disponível em: https://www.dadosefatos.turismo.gov.br/gloss\%C3\%A1rio-do-turismo/884-c.html Acesso em: 05 jun. 2019.

BRUHNS, H. (Org). Introdução aos estudos do lazer. Campinas: Editora da Unicamp, 1997.

BUGDAY, E. B.; LEMBET, Z. A New Perspective in Marketing: Entertainment Marketing. International Journal of Innovative Research and Development, Kolkata, v. 5, n. 6, p. 1-8, 2016.

CAETANO, M.L.S. et al. Clareza, atualização, acesso às informações e estética em sites de Organizações Não Governamentais. Research Society and Development, Itabira, v.2, n.1, p. 80-92. 2016

COSTA, A.M.J.F.; VIDOTTI, S.A.B.G.A. Encontrabilidade da Informação em Web Sites de Museus. Informação E Profissões, Londrina, v.5, n.2, p.79 -101, 2017.

DENECKE, K.; DENG, Y. Sentiment analysis in medical settings: new opportunities and challenges. Artificial Intelligence in Medicine, Netherlands, v. 64, n.1, p. 1727, 2015.

DHAMI, I.; DENG, J. Linking the recreation opportunity spectrum with travel spending: a spatial analysis in West Virginia. Leisure Sciences, Birmingham, v.1, n.1, p.1-15, 2017.

DIAS, J.D. Desenvolvimento de serious game para auxílio ao enfrentamento da 
obesidade infantil. 137f. Dissertação (Mestrado em Ciências da Saúde): Programa de Pós-graduação em Enfermagem. Universidade Federal de São Carlos, São Carlos, 2015 .

DUMAZEDIER, J. Sociologia empírica do lazer. São Paulo: Perspectiva: SESC, 1979.

GUARIZO, M.A. Aspectos relevantes na sistematização das atividades recreativas: visão do profissional, $40 \mathrm{f}$. Trabalho de conclusão de curso (bacharelado - Educação Física) - Universidade Estadual Paulista, Instituto de Biociências de Rio Claro, 2015.

GUERRA, M. Recreação e Lazer. Porto Alegre: Sagra, 1985

HUSSAIN, D.; ROSS, P.; BEDNAR, P. The Perception of the Benefits and Drawbacks of Internet Usage by the Elderly People. In: ROSSIGNOLI, C., VIRILI, F.; ZA, S (Eds). Digital technology and organizational change: reshaping technology, people, and organizations towards a global society. Cham: Springer, 2018. cap.10, p 199-212.

JACOSKI, C. A.; DALLACORTE, C.; MOTA, A. E. R. Aplicação de uma pesquisa de usabilidade para reestruturação do layout da página web infohab. e-Revista LOGO, Florianópolis, v.6, n.1, p. 85-101, 2017.

JAHREN, P.; SUI, T. How water influences our lives. Singapura: Springer Singapore, 2017.

JONGSTRA S.; BEISHUIZEN C.; ANDRIEU S.; et al. Development and Validation of an Interactive Internet Platform for Older People: The Healthy Ageing Through Internet Counselling in the Elderly Study. Telemedicine and e-Health, New Rochelle, v.23, n.2, p. 96-104, 2017.

LAZAR, J.; OLALERE, A.; WENTZ, B. Investigating the accessibility and usability of job application web sites for blind users. Journal of Usability Studies, New York, v.7, n.2, p. 68-87, 2012.

LEE, SY. Analysis of relationship marketing factors for sports centers with mixed methods research. Asia Pacific Journal of Marketing and Logistics, West Yorkshire, v.30, n.1, p. 182-197, 2018.

LIMEIRA, C.F.D. Avaliação, análise e desenvolvimento de jogo sério digital para desktop sobre sintomas e procedimentos de emergência do Acidente Vascular Cerebral. 147f. Dissertação (Mestrado em Ergodesign) - Programa de Pós-graduação em Design. Universidade Federal do Rio Grande do Norte, Rio Grande do Norte, 2015.

LONGARAY, A. A. et al. Usando informações para medir a análise de satisfação dos serviços prestados por sites de e-commerce. Biblos - Revista do Instituto de Ciências Humanas e da Informação, Rio Grande, v.30, n.2, p. 22-51, 2017.

MARASCO, A. et al. Exploring the role of next-generation virtual technologies in destination marketing. Destination Marketing \& Management, Kidlington, v.7, n.1, 
p.1-14.

MARTEL, F. Smart: uma pesquisa sobre as internets. Revista Rumores. São Paulo, v.10, n.20, p. 7-25, 2017.

NIELSEN, J. Designing Web Usability. Indiana: New Riders Publishing, 2000.

Designing web usability: The practice of simplicity. São Francisco: New Riders Publishing, 1999.

NORONHA, D.F.; RIBEIRO, A.J.P. Atividades lúdicas nas aulas de educação física: contribuições para aquisição/manutenção de hábitos saudáveis. DO CORPO: Ciências e Artes, Caxias do Sul, v.7, n.1, p.61-86. 2017.

NUNES M.B. Running Weel: proposta e análise de um exergame motivacional para corrida. 132f. Dissertação. (Mestrado em Ciências da Computação) - Departamento de Informática. Universidade Federal do Rio Grande do Sul, Rio Grande do Sul, 2014.

OLIVEIRA, L.A.P. Ressonâncias da Tecnologia Virtual na Recreação em Hotéis. f.83. Dissertação (Mestrado em Desenvolvimento Humano e Tecnologia) - Programa de Pós- Graduação em Desenvolvimento Humano e Tecnologias, Universidade Estadual Paulista, Rio Claro, 2017.

PAIM, C.A. Octopus: um modelo de gamification para auxílio no cuidado ubíquo de doenças crônicas não transmissíveis. 84f. Dissertação (Mestrado em Computação) Programa de Pós- graduação em Computação Aplicada, Universidade do Vale do Rios dos Sinos, São Leopoldo, 2015.

PAZ, F. et al. Perception Study of a New Set of Usability Heuristics for Transactional Web Sites. In: INTELLIGENT HUMAN SYSTEMS INTEGRATION (IHSI), 2018, Cham, Proceedings ..., Cham: Springer, 2018, p.620-625.

PEREIRA, A.S. et al. Ludicidade e meio ambiente: uma proposta de socialização do pibid-educação do campo. In: XI CONGRESSO NACIONAL DE EDUCAÇÃO, 2013, Curitiba. Anais..., Curitiba: Educere, 2013, p.1-11.

PINTO, M.M. A Gestão da Informação nas Universidades Públicas portuguesas: reequacionamento e proposta de modelo. Tese (Doutoramento em Informação e Comunicação em Plataformas Digitais) - Programa de Informação e Comunicação em Plataformas Digitais. Universidade do Porto. Porto, 2015.

RICHARDSON, R.J. Pesquisa social: métodos e técnicas. 4. ed. São Paulo: Atlas, 2017.

RODRIGUES M.H. Criação, desenvolvimento e aplicação de serious game educativo para a prevenção em saúde bucal infantil - "Caí, Perdi um Dente... E daí?”. 153f. Dissertação (Mestrado em Ciências Odontológicas): Programa de Ciências Odontológicas aplicadas. Universidade de São Paulo, Bauru, 2014.

RODRIGUES, F. N.; DANTAS, E. W. C. resorts no Nordeste brasileiro: da inserção ao discurso empresarial verde no porto das dunas, Aquiraz/CE. Geosaberes, 
Fortaleza, v. 8, n. 15, p. 2-20, 2017.

RODRÍGUEZ, G.; PÉREZ, J.; CUEVA, S.; TORRES, R. A framework for improving web accessibility and usability of Open Course Ware sites. Computers \& Education, Amsterdam, v. 109, n. 1, p. 197-215, 2017.

SCHNEIDER, M.D.; ZILLI, J.C.; VIEIRA, A.C.P. Diagnóstico mercadológico: um estudo em uma vinícola nos Vales da Uva Goethe - Santa Catarina. Revista da Universidade Vale do Rio Verde, Três Corações, v. 15, n. 2, p.744-750, 2017.

SCHWARTZ, G. M.; SANTIAGO, D. R. P. ; N.; TAVARES, G. H. ; FIGUEIREDO, J. P. ; NAZARIO, M. E. S. . Gestão da informação sobre esporte recreativo e lazer: Balanço da Rede CEDES. Jundiaí: Fontoura, 2010.

SELLER, M.L.; LAURINDO, F.J.B. Comunidade de marca ou boca a boca eletrônico: qual o objetivo da presença de empresas em mídias sociais? Gestão \& Produção. São Carlos, v. 25, n. 1, p. 191-203, 2018.

SIMÃO, S.J.P.; ANDRADE, M.; GOMES, M; et al. Análise da usabilidade da homepage da Apple Brasil. In: Blucher Engineering Proceedings, 2016, Recife, Proceedings... São Paulo: Blucher, 2016, p.1-13.

SOUZA, E.A. et al. Review of the Use of Information Technology in Brazilian Schools from 2010 to 2014. International Journal of Information and Education Technology, Pittsburgh, v.7, n.4, p. 284-290, 2017.

SPARAPANI, V.C. Um jogo feito pra mim: estrutura conceitual para o desenvolvimento de videogames para crianças com diabetes mellitus tipo 1. 299f. Tese (Doutorado em Ciências) Programa de Pós-graduação Enfermagem em Saúde Pública. Universidade de São Paulo, Ribeirão Preto, 2015.

SUTTON, H. Follow 5 steps to create elegant and accessible design. Disability Compliance for Higher Education, Hoboken, v.22, n.6, p.1-5. 2017

TORRANCE, H. Experimenting with qualitative inquiry. Qualitative Inquiry, New Castle, v. 23, n. 1, p. 69-76, 2017.

URIBE, S.; ÁLVAREZ, F.; MENÉNDEZ, J.M. User's Web Page Aesthetics Opinion: A Matter of Low-Level Image Descriptors Based on MPEG-7. ACM Transactions on the Web, New York, v.11, n b.1, p. 1-25, 2017.

VAGHETTI, C.A.O. Exergames em rede: a educação física no cyberspace. $184 \mathrm{f}$. Tese (Doutorado em Ciências) Programa de Pós-graduação em Ciências. Universidade Federal do Rio Grande, Rio Grande do Sul, 2013.

VIVEKANANTHAM, A. et al. Evaluating on-line health information for patients with polymyalgia rheumatica: a descriptive study. BMC musculoskelet (Online), Montgomery, v.18, n.1, p. 43-50. 2017.

ZHANG, H., CHOI, Y.K. Preannouncement messages: impetus for electronic wordof- outh. International Journal of Advertising, Abingdon, v.37, n. 1, p. 54-70, 2018. 


\section{Endereço das Autoras:}

Marília Amábile Guarizo

Rua Claudio Gilmar Guidi, n 39, Casa 2 - Três Pontes

Amparo - SP - 13.909-030

Endereço Eletrônico: mah_guarizo@hotmail.com

Gisele Maria Schwartz

Av. 24 A, n 1515 - Bela Vista

Rio Claro - SP - 13.506-900

Endereço Eletrônico: schwartz@rc.unesp.br 